\title{
Conditions for Preparing Glycyrrhiza uralensis Extract for Inhibiting Biofilm Formation of Streptococcus mutans ${ }^{1}$
}

\author{
Youngseok $\mathrm{Ham}^{2} \cdot$ Tae-Jong Kim (D) ${ }^{2, \dagger}$
}

\begin{abstract}
Licorice, which has an extensive history of use as an herbal medicine, has been suggested to have oral health benefits. However, to date, no systematic study has been conducted on the preparation method of licorice extracts for oral health. In this study, licorice extracts prepared using water and ethanol were investigated for its ability to inhibit the biofilm formation of Streptococcus mutans. The licorice extract prepared with around $60 \%$ ethanol effectively inhibited the biofilm formation of $S$. mutans. Licorice extracted with $50 \%$ ethanol almost completely inhibited the biofilm formation at $1.5 \mathrm{~g} / \mathrm{L}$ of licorice extract. This inhibitory activity was confirmed in a microplate assay and a flow cell system. Glycyrrhetic acid was extracted from licorice effectively with $60 \%$ ethanol concentration. The strong inhibitory activity of glycyrrhetic acid and the synergistic inhibition with glycyrrhizin on biofilm formation were suggested as major reasons for a concentration-specific extraction. These results suggest that licorice extract prepared using around $60 \%$ ethanol effectively inhibits the biofilm formation of $S$. mutans.
\end{abstract}

Keywords: licorice, ethanol extract, biofilm, Streptococcus mutans, glycyrrhetic acid

\section{INTRODUCTION}

Licorice, the root and rhizome of Glycyrrhiza sp., has been traditional used as a medicinal herb and a natural sweetener. It prevents gastrointestinal ulcers (Momeni et al., 2014; Jalilzadeh-Amin et al., 2015); neutralizes toxic properties of compounds (Isbrucker and Burdock, 2006; Gong et al., 2015); and has antiviral (Wang et al., 2013; Fukuchi et al., 2016), antibiotic (He et al., 2006; Gupta et al., 2008; Lau and Plotkin, 2013; Chakotiya et al., 2016; Rohinishree and Negi, 2016), antifungal (Messier and Grenier, 2011; Seleem et al., 2016; Sharma et al., 2016), and anticancer activities (Shen et al., 2015; Fukuchi et al., 2016). Over the past 20 years, the number of studies on the use of licorice for oral health has steadily increased. Compounds in licorice improve bad breath by reducing the bacterial production of volatile sulfur compounds (Shin-ichi et al., 2012). Licorice has also been shown to ameliorate mouth dryness more effectively than water (Yu et al., 2016). In particular, licorice is thought to enhance oral health by inhibiting the growth of Streptococcus mutans, a primary causative agent of tooth decay (Segal et al., 1985; Hwang et al., 2004; Jain et al., 2013; Ajagannanavar et al., 2014). Consequently, the licorice lollipop was developed to control dental

\footnotetext{
${ }^{1}$ Date Received August 21, 2018, Date Accepted February 21, 2019

2 Department of Forest Products and Biotechnology, Kookmin University, Seoul 02707, Republic of Korea

† Corresponding author: Tae-Jong Kim (e-mail: bigbell@kookmin.ac.kr, ORCID: 0000-0002-7483-0432)
} 
caries (Hum et al., 2011; Almaz et al., 2017).

Oral bacteria produce biofilms, called plaque, in the oral cavity. The oral cavity is a continuous flow system of food, drink, and saliva, and therefore, oral bacteria would be easily washed away without the ability to form a biofilm. Nonetheless, the oral cavity is a favorable environment for bacterial growth, and its many surfaces are coated with a plethora of bacteria, with >500-700 bacterial strains residing in the oral cavity (Rosan and Lamont, 2000; Dewhirst et al., 2010; Huang et al., 2011). Some of these bacteria are implicated in oral diseases. Moreover, specific oral bacteria strains have been associated with several systemic diseases. $S$. mutans, a gram-positive bacterium, is a regular member of the mature dental biofilm community. S. mutans has four serotypes c, e, f, and k based on its polysaccharides. Almost $70-80 \%$ of $S$. mutans found in the oral cavity is serotype c. However, under certain conditions, $S$. mutans can become prevalent and cause dental caries (Loesche, 1986; Ahn et al., 2008).

S. mutans produces insoluble glucan using sugars present in food. The insoluble glucan aids in plaque formation on the tooth surface where $S$. mutans exists (Krzysciak et al., 2014; Ren et al., 2016). S. mutans in the plaque cannot be easily removed by physical and chemical treatments (Welin-Neilands and Svensater, 2007; Bowen and Koo, 2011). The plaque also facilitates the intraoral adhesion of other bacteria, and these bacteria have been reported to cause not only oral diseases but also other complications, including endocarditis (Berbari et al., 1997), pneumonia (Scannapieco, 1999), low birth weight (Buduneli et al., 2005), and cardiovascular disease (Beck et al., 1996). These previous studies have suggested that chemical inhibition of biofilm formation in the oral cavity may play an important biological role in human health. One way to inhibit biofilm formation is by killing bacterial cells (Kondo et al., 2007). Recently, plant extracts that inhibited biofilm formation without the bactericidal effect of $S$. mutans have also been reported (Ham and Kim, 2018).

Although many studies have reported the beneficial effects of licorice extracts on oral health, no systematic study has been conducted on the extraction methods for preparing licorice extracts for inhibiting the biofilm formation of $S$. mutans. In this study, the activity of licorice extract to inhibit the biofilm formation of $S$. mutans was investigated according to ethanol concentration of extraction solvent. In addition, the extraction of the chemicals inhibiting the biofilm formation according to the ethanol concentration was observed to explain the specific extraction conditions.

\section{MATERIALS and METHODS}

\subsection{Media for cell culture and biofilm formation assays}

Streptococcus mutans serotype c was obtained from LG Household \& Health Care Ltd (Daejeon, Korea). Brain heart infusion (BHI) broth (BD Biosciences Korea, Seoul, Korea) was used for growing $S$. mutans. BHI agar was prepared by adding $1.5 \%$ agar to $\mathrm{BHI}$ broth. BHI-S medium, the biofilm formation medium, was prepared by supplementing BHI broth with 10-g/L sucrose. Glycyrrhizin (product number: G0150), glycyrrhetic acid (product number: G0149), and glucuronic acid (product number: G0302) were purchased from Tokyo Chemical Industry Co., Ltd. (Tokyo, Japan). Liquiritin was purchased from Kono Chemical Co., Ltd. (Xian, China). Glycyrrhizin was dissolved in 50\% ethanol, liquiritin and glycyrrhetic acid were dissolved in dimethyl sulfoxide, and glucuronic acid was dissolved in distilled water before use, respectively.

\subsection{Preparation of licorice extracts}

Licorice was purchased from Jiundang Oriental 
Medicine Co. (Seoul, Korea). The dried licorice extracts were prepared using licorice pulverized to a diameter of $\leq 1 \mathrm{~mm}$. Subsequently, $10 \mathrm{~g}$ of the powdered licorice were mixed with $50-\mathrm{mL}$ of water and ethanol and incubated at $50^{\circ} \mathrm{C}$ for $3 \mathrm{~h}$. The content of ethanol in the extract solution was indicated in each experiment. The residual solid materials were removed by filtration (Whatman filter paper Grade 1). The filtrates were concentrated using a rotary evaporator (RV10, IKA ${ }^{\circledR}$ Korea, Ltd., Seoul, Korea). Without making powder, the original volume of the pre-concentrate extract was restored by adding distilled water. Insoluble materials were removed by centrifugation at $16,810 \times g$ for 20 min and filtration through a syringe filter (25-mm diameter, cellulose acetate, $0.20-\mu \mathrm{m}$ pore size; GVS North America, Inc., Sanford, ME, USA). The transparent extracts were stored at $-80^{\circ} \mathrm{C}$.

\subsection{Biofilm formation assay and quantitative analysis}

S. mutans, which was stored at $-80^{\circ} \mathrm{C}$, was streaked onto a $\mathrm{BHI}$ agar plate and cultured at $37^{\circ} \mathrm{C}$ for 2 days. A single colony was inoculated into 5-mL BHI broth and incubated at $37^{\circ} \mathrm{C}$ for $24 \mathrm{~h}$ without shaking. The cell density of cultured $S$. mutans was measured at an absorbance of $600 \mathrm{~nm}\left(\mathrm{Abs}_{600}\right)$ using an Optizen 2120 UV Plus spectrophotometer (Mecasys Co., Ltd., Daejeon, Korea). A 96-well polyvinyl chloride microplate containing $100-\mu \mathrm{L}$ BHI-S medium was inoculated with the cultured cells at an optical density of 0.05 at $\mathrm{Abs}_{600}$. After incubation at $37^{\circ} \mathrm{C}$ for $24 \mathrm{~h}$, the cell density was measured at $\mathrm{Abs}_{595}$ using an Opsys MR microplate reader (Dynex Technologies, Chantilly, VA, USA). After removing planktonic cells with distilled water, $100 \mu \mathrm{L}$ of $1 \%$ crystal violet solution was dispensed into each well and allowed to stand for $15 \mathrm{~min}$. After washing gently three times with distilled water, 100 $\mu \mathrm{L}$ of $95 \%$ ethanol was added to each well. After incubating for $15 \mathrm{~min}$ to allow the elution of the stained crystal violet, $\mathrm{Abs}_{595}$ was measured using an Opsys MR microplate reader. The one-way analysis of variance was used to distinguish differences from controls at the 95\% confidence level by Tukey test using SPSS software (Ver. 23.0, SPSS Inc., Chicago, Illinois, USA).

\subsection{Biofilm formation in a flow cell system}

To observe biofilm formation in a flow state similar to the oral cavity, biofilm formation of $S$. mutans was monitored in a continuous flow cell system. The flow cell system was purchased from the Center for Biomedical Microbiology (Technical University of Denmark, Lyngby, Denmark). The flow cell channel was covered with a polyvinyl chloride coverslip on which a biofilm was formed. Licorice extracted with $50 \%$ ethanol was added to the BHI-S medium at the indicated concentration.

As a preculture, S. mutans was inoculated into 5-mL BHI broth and incubated at $37^{\circ} \mathrm{C}$ for $24 \mathrm{~h}$ without shaking. The precultured cells were diluted to an optical density of 0.05 at $\mathrm{Abs}_{600}$, and $350 \mu \mathrm{L}$ of diluted cells were inoculated into each channel. The coverslip was placed facing downward for $1 \mathrm{~h}$, giving the cells a chance to attach to the surface of the coverslip. The flow cell system was turned on with the coverslip pointing upward and cultured for $24 \mathrm{~h}$ at a constant flow rate of $13 \mathrm{~mL} / \mathrm{h}$. The biofilm medium was continuously supplied to the flow cell system using a peristaltic pump (Masterflex L/S Digital Drive EW07523-90, Masterflex L/S 12-channel, 8-roller cartridge pump head EW-07519-25, Masterflex L/S small cartridges EW-07519-85, Cole-Parmer Instrument Company, LLC., Vernon Hills, IL USA). The biofilm formed under the coverslip was observed using an Axio Scope.A1 microscope (Carl Zeiss Co., Ltd., Seoul, Korea) equipped with ZEN microscope software (Carl Zeiss Co., Ltd). 


\subsection{Analysis of glycyrrhizin and glycyrrhetic acid in licorice extract using high performance liquid chromatograph}

For analysis of glycyrrhizin and glycyrrhetic acid in licorice extracts, the method of a previous study (Wang and Yang, 2007) has been used. The equipment of high performance liquid chromatograph was Acme 9000 (YL Instruments Co., Ltd, Anyang, Korea). The analytical column used was YMC-Triart C18 (Product code: TA12S05-2546WT, YMC Korea Co., Ltd, Seongnam, Korea). The flow rate of mobile phase was $1.2 \mathrm{~mL} / \mathrm{min}$. The wavelength of light for UV detector was $254 \mathrm{~nm}$. The column temperature was $40^{\circ} \mathrm{C}$. The injection volume of sample was $10 \mu \mathrm{L}$. The licorice extract was separated using a gradient mobile phase according to a previous study (Wang and Yang, 2007). The data were analyzed with Autochro-3000 software (version 2.0.0).

\section{RESULTS and DISCUSSION}

\subsection{Ethanol content of extract solvents for inhibited biofilm formation of Streptococcus mutans}

Licorice has recently been shown to be effective against dental caries and oro-dental diseases (Touyz, 2009; Messier et al., 2012). In previous studies, various solvents were used for licorice extraction (He et al., 2006; Al-Turki et al., 2008; Nitalikar et al., 2010; Ahn et al., 2012; Rodino et al., 2015; Karahan et al., 2016). In the present study, water and ethanol were tested to prepare the licorice extract for evaluating its inhibitory activity on the biofilm formation of $S$. mutans. Biofilm formation and cell growth inhibition were measured as a function of the ethanol content in the extracting solvent (Fig. 1). When $5 \%$ by volume of the extract was treated with the culture, a robust
A.

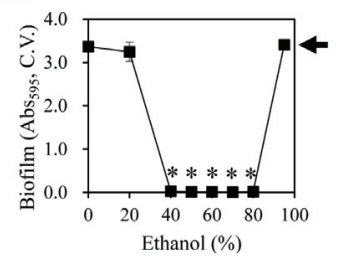

C.

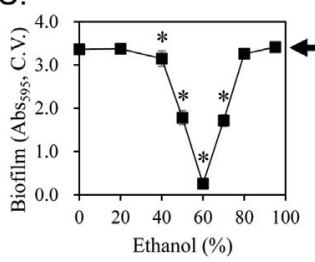

B.

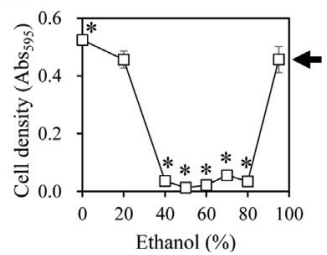

D.

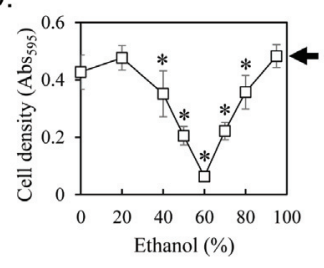

Fig. 1. Effect of ethanol content of extract solvent on the biofilm formation (A and $\mathrm{C}$ ) and the cell growth (B and D) of S. mutans. Extracts of 5\% (A and B) and $1.25 \%$ (B and $\mathrm{C}$ ) of the tested culture volume were treated. The control value is the result of the untreated extract, which is indicated on the right side of the figure by a black arrow. The standard deviation was calculated from five independent experimental results. Statistical analysis was performed using a tukey test. Values that differ from the control with the $95 \%$ confidence level are marked with a star on the top of symbols.

inhibitory effect on biofilm formation was observed using licorice extracts made with $40 \%-80 \%$ ethanol (Fig. 1A). The cell growth of $S$. mutans was correlated with biofilm formation (Fig. 1B). To determine the range of effective ethanol concentrations more accurately, we tested with extracts having $1.25 \%$ of the cell culture volume tested (Fig. 1C and 1D). Clearly, the most effective inhibitory activity on the biofilm formation of $S$. mutans was shown the licorice extract made with $60 \%$ ethanol.

To confirm the effect of ethanol content in the extracting solvent in Fig. 1, the amount of the solvent required for inhibiting the biofilm formation of $S$. mutans was examined (Fig. 2). The licorice extracts prepared using water or $95 \%$ ethanol as the extraction solvent showed no inhibitory effect on biofilm 
A.

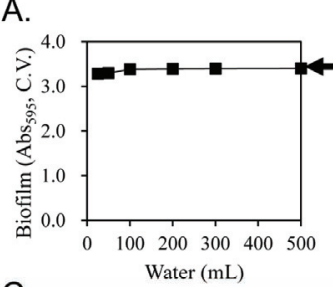

C.

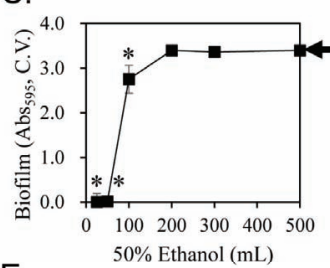

E.

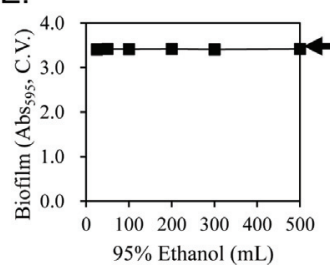

B.

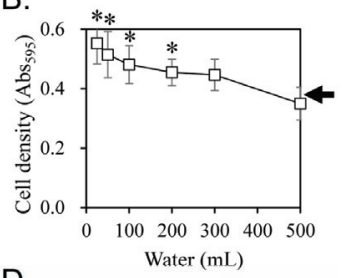

D.

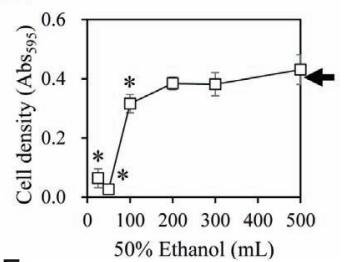

F.

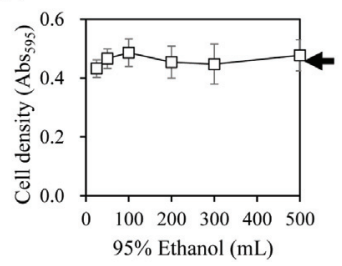

Fig. 2. Effect of the licorice-to-solvent volume ratio on the biofilm formation (A, C, and E) and the cell growth $(\mathrm{B}, \mathrm{D}$, and $\mathrm{F}$ ) of $S$. mutans. The inhibitory activity of extracts with $5 \%$ of tested cell culture volume was evaluated according to the volume of extract solvent: water (A and B), 50\% ethanol (C and $D$ ), and $95 \%$ ethanol ( $E$ and $F$ ). The control value is the result of the untreated extract, which is indicated on the right side of the figure by a black arrow. The standard deviation was calculated from five independent experimental results. Statistical analysis was performed using a tukey test. Values that differ from the control with the $95 \%$ confidence level are marked with a star on the top of symbols.

formation, irrespective of the amount of solvent (Fig. $2 \mathrm{~A}$ and $2 \mathrm{E}$, respectively). In case of $50 \%$ ethanol, the inhibitory activity on biofilm formation started appearing using the licorice extract prepared with 100-mL solvent and most biofilm formation was inhibited by licorice extracts prepared with a solvent volume of less than 50-mL (Fig. 2C). The effect of the licorice extract on the cell growth of $S$. mutans
Table 1. Yield of licorice extract according to ethanol content in extract solvent.

\begin{tabular}{cccc}
\hline $\begin{array}{c}\text { Ethanol content } \\
\text { in extract solvent } \\
(\%)\end{array}$ & $\begin{array}{c}\text { Solid yield } \\
\text { in extract } \\
(\%)^{\mathrm{a}}\end{array}$ & $\begin{array}{c}\text { Glycyrrhizin } \\
(\mathrm{g} / \mathrm{L})\end{array}$ & $\begin{array}{c}\text { Glycyrrhetic } \\
\text { acid (g/L) }\end{array}$ \\
\hline 0 & 23.9 & 5.10 & 0.001 \\
20 & 24.5 & 6.51 & 0.006 \\
40 & 23.9 & 6.91 & 0.019 \\
50 & 24.2 & 7.42 & 0.046 \\
60 & 23.7 & 6.95 & 0.027 \\
70 & 22.6 & 6.48 & 0.024 \\
80 & 17.7 & 4.53 & 0.019 \\
95 & 5.9 & 0.37 & 0.000 \\
\hline
\end{tabular}

\footnotetext{
${ }^{\text {a }}$ Solid yield (\%) = total weight of solid in extract (g) / weight of licorice used for extraction $(g) \times 100$
}

was comparable to that on biofilm formation (Fig. 2D and $2 \mathrm{~F}$ ), except for the water extract (Fig. 2B). The results in Fig. 2 confirmed that licorice extracted with around $50 \%$ aqueous ethanol effectively inhibited the biofilm formation of $S$. mutans in Fig. 1. The yield in extraction was measured according to the ethanol content (Table 1). There was no significant change in the solid yield up to $70 \%$ ethanol, but it decreased rapidly at more than $80 \%$ ethanol. This result support that there was a large portion of water soluble materials in licorice (Kondo et al., 2007). However, the specific inhibitory effect on the biofilm formation shown in the licorice extract using $60 \%$ ethanol as the extraction solvent cannot be explained by the yield of the extract.

The inhibitory effect on biofilm formation was not observed using the water extract, which is the traditional method for making licorice extract, or the $95 \%$ ethanol extract. The biofilm formation of S. mutans was inhibited by extracts prepared with $60 \%$ ethanol content. Considering that ethanol has polarity like water, this ethanol concentration-specific extraction condition is highly unusual. 
A.

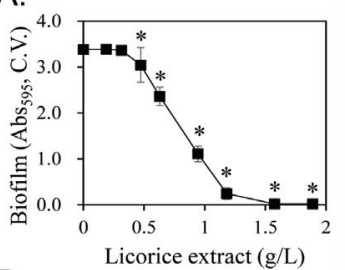

B.

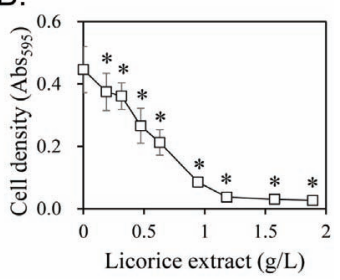

Fig. 3. Effect of extract amount on the biofilm formation and the cell growth of $S$. mutans. The biofilm formation (A) and cell growth (B) were evaluated using a microplate assay. The control value is the result at $0 \mathrm{~g} / \mathrm{L}$ in each figure. The standard deviation was calculated from five independent experimental results. Statistical analysis was performed using a tukey test. Values that differ from the control with the 95\% confidence level are marked with a star on the top of symbols.

\subsection{Inhibitory activity of licorice extracted with $50 \%$ ethanol on biofilm formation}

The inhibitory activity of licorice on the biofilm formation of $S$. mutans was evaluated in a microplate assay and in a flow cell system using the $50 \%$ aqueous ethanol licorice extract (Fig. 3 and Fig. 4, respectively).
Biofilm formation was inhibited by $0.5-\mathrm{g} / \mathrm{L}$ licorice extract, and most of the biofilm formation was inhibited at concentrations around $1.5 \mathrm{~g} / \mathrm{L}$ (Fig. 3A). Cell growth was correlated with the biofilm formation (Fig. 3B), as observed in Fig. 1.

The inhibitory effect of licorice extract on the biofilm formation of $S$. mutans was confirmed using a continuous flow cell system (Fig. 4). Consistent with the microplate experiments, the inhibitory activity on the biofilm formation began to appear at $0.5-\mathrm{g} / \mathrm{L}$ licorice extract.

\subsection{Synergistic inhibition of glycyrrhizin and glycyrrhetic acid on the biofilm formation of $S$. mutans}

Previous studies on the treatment of oral diseases using licorice have suggested that licorice may kill $S$. mutans cells directly (Ajagannanavar et al., 2014; Wang et al., 2015) and/or inhibit plaque formation (Segal et al., 1985; Ahn et al., 2012). Several components of licorice have been reported to exhibit physiological functions that kill oral microorganisms (He et al., 2006; Kondo et al., 2007). We also evaluated the inhibitory

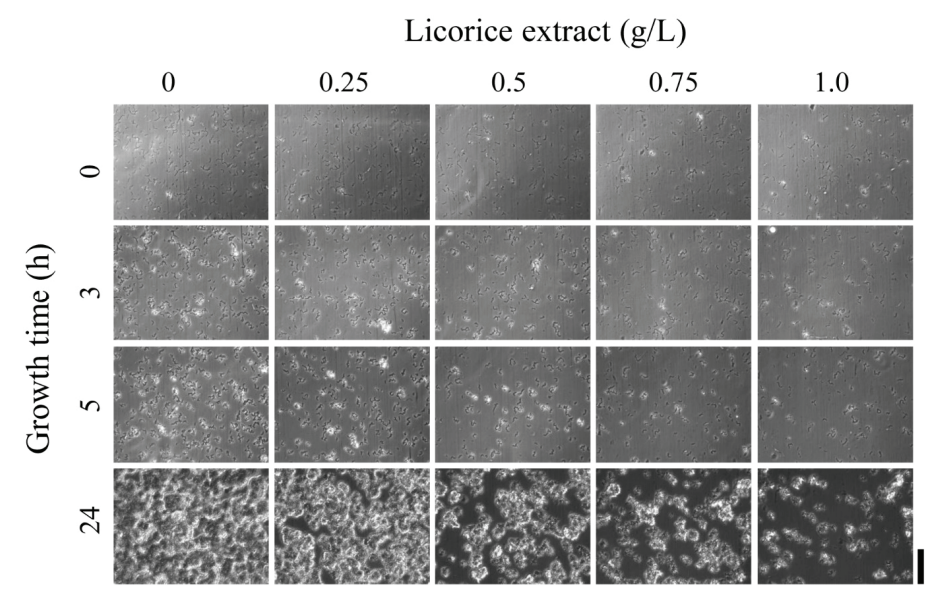

Fig. 4. Effect of licorice extract on the biofilm formation of $S$. mutans in a flow cell chamber. The microscope magnification was $\times 400$. Scale bar on the bottom right of figure indicates $50 \mu \mathrm{m}$. 
A.
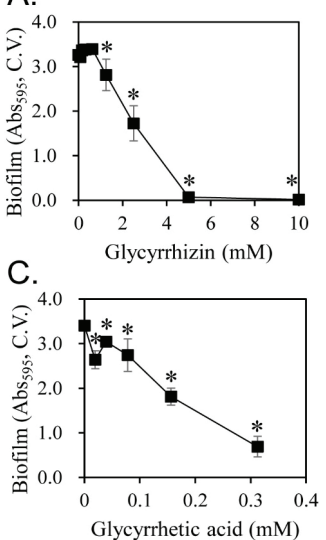

E.
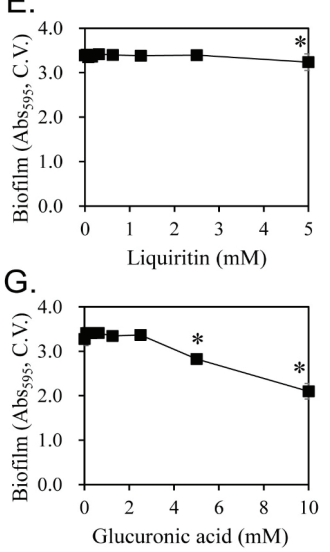

B.
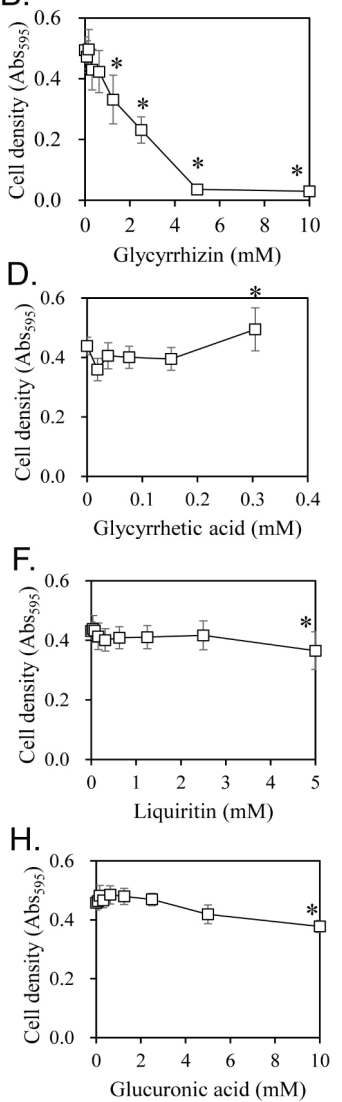

Fig. 5. Effect of glycyrrhizin, glycyrrhetic acid, liquiritin, and glucuronic acid on the biofilm formation (A, C, $\mathrm{E}$, and $\mathrm{G}$ ) and the growth inhibition (B, D, F, and $\mathrm{H}$ ) of $S$. mutans. The control value is the result at $0 \mathrm{~g} / \mathrm{L}$ in each figure. The standard deviation was calculated from five independent experimental results. Statistical analysis was performed using a tukey test. Values that differ from the control with the 95\% confidence level are marked with a star on the top of symbols.

activity of the main components of licorice on biofilm formation (Fig. 5). Glycyrrhizin and glycyrrhetic acid showed strong inhibitory activity, but liquiritin and glucuronic acid had no and weak inhibitory activity, respectively. Therefore, the content of glycyrrhizin and glycyrrhetic acid in extracts were analyzed according to the ethanol concentration of the extract solvent (Table

1). Glycyrrhizin is extracted at high concentration in water and 50\% ethanol and at very low concentration in 95\% ethanol. Therefore, glycyrrhizin, a major component of licorice, cannot explain the specific strong biofilm formation inhibition in $60 \%$ ethanol extracts. In the case of glycyrrhetic acid, it was extracted at $0.001 \mathrm{~g} / \mathrm{L}$ by water. However, $50 \%$ ethanol extracts glycyrrhetic acid at $0.046 \mathrm{~g} / \mathrm{L}$, which is 46 times higher than water extract. Glycyrrhetic acid was not detected in the $95 \%$ ethanol extract. This can partially explain the specific strong biofilm formation inhibition in the $60 \%$ ethanol extract. In Fig. 5, the inhibitory activity of glycyrrhetic acid on biofilm formation is around ten times stronger than that of glycyrrhizin. However, the content of glycyrrhetic acid in the 50\% ethanol extract is less than $1 \%$ of glycyrrhizin, and it is not enough amount to explain the specific strong biofilm formation inhibition in the $60 \%$ ethanol extract, despite 10 -fold strong inhibitory activity on biofilm formation.

In order to explain the specific strong biofilm formation inhibition in the $60 \%$ ethanol extract, the synergistic inhibitory effect of glycyrrhizin and glycyrrhetic acid was evaluated (Fig. 6). When cells were treated with a mixture of $1.25 \mathrm{mM}$ glycyrrhizin and $0.075 \mathrm{mM}$ glycyrrhetic acid, which had no inhibitory activity individually, about $62 \%$ of biofilm formation was inhibited. When glycyrrhizin or glycyrrhetic acid was treated at concentrations of 2.5 $\mathrm{mM}$ or $0.15 \mathrm{mM}$, respectively, which were twice the treatment concentration, both chemicals showed around $47 \%$ inhibition of biofilm formation (Fig. 5). The inhibitory activity of the two compound mixture, 62\%, shows a synergistic effect, compared with $47 \%$, which is the inhibitory activity of treating each compound with a double concentration. Considering both a strong inhibitory activity of glycyrrhetic acid and its synergistic effect with glycyrrhizin on the biofilm formation of S. mutans, ethanol concentration specific extraction for glycyrrhetic acid may be a major reason for the strong 

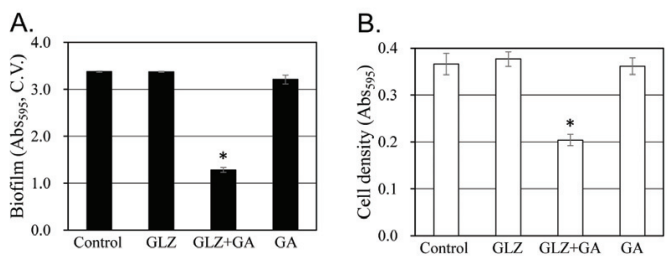

Fig. 6. Synergistic inhibition of a mixture of glycyrrhizin and glycyrrhetic acid on the biofilm formation (A) and the cell growth (B) of S. mutans. The concentrations were $1.25 \mathrm{mM}$ for glycyrrhizin (GLZ) and $0.075 \mathrm{mM}$ for glycyrrhetic acid (GA). The control value is the result of $5 \%$ treatment of DMSO, a solvent in which the compound is dissolved, without any compound. The standard deviation was calculated from five independent experimental results. Statistical analysis was performed using a tukey test. Values that differ from the control with the $95 \%$ confidence level are marked with a star on the top of symbols.

inhibitory activity of $60 \%$ ethanol extracts of licorice.

Inhibition of biofilm formation mediated by licorice could be attributed to the prevention of bacterial growth. Previous studies have shown that glycyrrhizin from licorice inhibits biofilm formation of $S$. mutans by inhibiting or killing the cell growth (He et al., 2006; Kondo et al., 2007). However, in this study, licorice extract with $60 \%$ ethanol is more effective when considering only inhibition of biofilm formation. This ethanol concentration specific extracts can be explained by the specific ethanol concentration extraction for glycyrrhetic acid from licorice and its synergetic inhibitory effect with glycyrrhizin. In order to maximize the effect of inhibiting the formation of plaque, a biofilm of $S$. mutans causing tooth decay, the extraction conditions of licorice were suggested and the corresponding chemical, glycyrrhetic acid, was presented in this study. In addition to the antioxidant activities (Nam et al., 2018; Kim et al., 2017; Kim et al., 2018), inhibition of microbial biofilm formation suggests new functionality of plant extracts.

\section{CONCLUSION}

Licorice is a traditional medicine with a sweet taste. Interestingly, it has been known that licorice is effective in preventing tooth decay. This study showed that licorice extract inhibits the cell growth and biofilm formation of $S$. mutans, which is a major cause of tooth decay. We found that licorice extracts prepared using around $60 \%$ ethanol effectively inhibited the biofilm formation of $S$. mutans, whereas those prepared using general extraction methods with water showed no effect on the biofilm formation. The ethanol concentration specific inhibitory ability of licorice extracts was due to the strong inhibitory activity of glycyrrhetic acid and its synergistic inhibitory activity with glycyrrhizin on the biofilm formation of $S$. mutans.

\section{ACKNOWLEDGMENT}

This work was supported by the "Cooperative Research Program for Agriculture Science and Technology Development (Project No. PJ01389603)" Rural Development Administration, Republic of Korea.

\section{REFERENCES}

Ahn, S.J., Ahn, S.J., Wen, Z.T., Brady, L.J., Burne, R.A. 2008. Characteristics of biofilm formation by Streptococcus mutans in the presence of saliva. Infection and Immunity 76(9): 4259-4268.

Ahn, S.J., Cho, E.J., Kim, H.J., Park, S.N., Lim, Y.K., Kook, J.K. 2012. The antimicrobial effects of deglycyrrhizinated licorice root extract on Streptococcus mutans UA159 in both planktonic and biofilm cultures. Anaerobe 18(6): 590-596.

Ajagannanavar, S.L., Battur, H., Shamarao, S., Sivakumar, V., Patil, P.U., Shanavas, P. 2014. Effect of aqueous and alcoholic licorice (Glycyrrhiza glabra) root extract against Streptococcus mutans 
and Lactobacillus acidophilus in comparison to chlorhexidine: An in vitro study. Journal of International Oral Health 6(4): 29-34.

Al-Turki, A.I., El-Ziney, M.G., Abdel-Salam, A.M. 2008. Chemical and anti-bacterial characterization of aqueous extracts of oregano, marjoram, sage and licorice and their application in milk and labneh. Journal of Food, Agriculture and Environment 6(1): 39-44.

Almaz, M.E., Sönmez, I.Ş., Ökte, Z., Oba, A.A. 2017. Efficacy of a sugar-free herbal lollipop for reducing salivary Streptococcus mutans levels: a randomized controlled trial. Clinical Oral Investigations 21(3): 839-845.

Beck, J., Garcia, R., Heiss, G., Vokonas, P.S., Offenbacher, S. 1996. Periodontal disease and cardiovascular disease. Journal of Periodontology 67(10 Suppl): 1123-1137.

Berbari, E.F., Cockerill, F.R., Steckelberg, J.M. 1997. Infective endocarditis due to unusual or fastidious microorganisms. Mayo Clinic Proceedings 72(6): 532-542.

Bowen, W.H., Koo, H. 2011. Biology of Streptococcus mutans-derived glucosyltransferases: Role in extracellular matrix formation of cariogenic biofilms. Caries Research 45(1): 69-86.

Buduneli, N., Baylas, H., Buduneli, E., Turkoglu, O., Kose, T., Dahlen, G. 2005. Periodontal infections and pre-term low birth weight: A case-control study. Journal of Clinical Periodontology 32(2): 174-181.

Chakotiya, A.S., Tanwar, A., Narula, A., Sharma, R.K. 2016. Alternative to antibiotics against Pseudomonas aeruginosa: Effects of Glycyrrhiza glabra on membrane permeability and inhibition of efflux activity and biofilm formation in Pseudomonas aeruginosa and its in vitro time-kill activity. Microbial Pathogenesis 98: 98-105.

Dewhirst, F.E., Chen, T., Izard, J., Paster, B.J., Tanner, A.C., Yu, W.H., Lakshmanan, A., Wade, W.G.
2010. The human oral microbiome. Journal of Bacteriology 192(19): 5002-5017.

Fukuchi, K., Okudaira, N., Adachi, K., Odai-Ide, R., Watanabe, S., Ohno, H., Yamamoto, M., Kanamoto, T., Terakubo, S., Nakashima, H., Uesawa, Y., Kagaya, H., Sakagami, H. 2016. Antiviral and antitumor activity of licorice root extracts. In Vivo 30(6): 777-785.

Gong, H., Zhang, B.-K., Yan, M., Fang, P.-F., Li, H.-D., Hu, C.-P., Yang, Y., Cao, P., Jiang, P., Fan, X.-R. 2015. A protective mechanism of licorice (Glycyrrhiza uralensis): Isoliquiritigenin stimulates detoxification system via Nrf2 activation. Journal of Ethnopharmacology 162: 134-139.

Gupta, V.K., Fatima, A., Faridi, U., Negi, A.S., Shanker, K., Kumar, J.K., Rahuja, N., Luqman, S., Sisodia, B.S., Saikia, D., Darokar, M.P., Khanuja, S.P.S. 2008. Antimicrobial potential of Glycyrrhiza glabra roots. Journal of Ethnopharmacology 116(2): 377380.

Ham, Y., Kim, T.-J. 2018. Plant extracts inhibiting biofilm formation by Streptococcus mutans without antibiotic activity. Journal of the Korean Wood Science and Technology 46(6): 692-702.

He, J., Chen, L., Heber, D., Shi, W., Lu, Q.-Y. 2006. Antibacterial compounds from Glycyrrhiza uralensis. Journal of Natural Products 69(1): 121-124.

Huang, R.J., Li, M.Y., Gregory, R.L. 2011. Bacterial interactions in dental biofilm. Virulence 2(5): 435-444.

Hum, C.-H., He, J., Eckert, R., Wu, X.-Y., Li, L.-N., Tian, Y., Lux, R., Shuffer, J.A., Gelman, F., Mentes, J., Spackman, S., Bauer, J., Anderson, M.H., Shi, W.-Y. 2011. Development and evaluation of a safe and effective sugar-free herbal lollipop that kills cavity-causing bacteria. International Journal of Oral Science 3(1): 13-20.

Hwang, J.-K., Shim, J.-S., Chung, J.-Y. 2004. Anticariogenic activity of some tropical medicinal 
Conditions for Preparing Glycyrrhiza uralensis Extract for Inhibiting Biofilm Formation of Streptococcus mutans

plants against Streptococcus mutans. Fitoterapia 75(6): 596-598.

Isbrucker, R.A., Burdock, G.A. 2006. Risk and safety assessment on the consumption of Licorice root (Glycyrrhiza sp.), its extract and powder as a food ingredient, with emphasis on the pharmacology and toxicology of glycyrrhizin. Regulatory Toxicology and Pharmacology 46(3): 167-192.

Jain, E., Pandey, R., Khanna, R. 2013. Liquorice root extracts as potent cariostatic agents in pediatric practice. The Journal of Indian Society of Pedodontics and Preventive Dentistry 31(3): 146152.

Jalilzadeh-Amin, G., Najarnezhad, V., Anassori, E., Mostafavi, M., Keshipour, H. 2015. Antiulcer properties of Glycyrrhiza glabra $L$. extract on experimental models of gastric ulcer in mice. Iranian Journal of Pharmaceutical Research 14(4): 1163-1170.

Karahan, F., Avsar, C., Ozyigit, I.I., Berber, I. 2016. Antimicrobial and antioxidant activities of medicinal plant Glycyrrhiza glabra var. glandulifera from different habitats. Biotechnology \& Biotechnological Equipment 30(4): 797-804.

Kim, J.-W., Um, M., Lee, J.-W. 2018. Antioxidant activities of hot water extracts from different parts of Rugosa rose (Rosa rugosa Thunb.). Journal of the Korean Wood Science and Technology 46(1): 38-47.

Kim, S.-H., Lee, S.-Y., Cho, S.-M., Hong, C.-Y., Park, S.-Y., Park, M.-J., Choi, I.-G. 2017. Antioxidant activities of Cryptomeria japonica leaves extracts by extraction methods. Journal of the Korean Wood Science and Technology 45(5): 495-510.

Kondo, K., Shiba, M., Nakamura, R., Morota, T., Shoyama, Y. 2007. Constituent properties of licorices derived from Glycyrrhiza uralensis, $G$. glabra, or $G$. inflata identified by genetic information. Biological and Pharmaceutical
Bulletin 30(7): 1271-1277.

Krzysciak, W., Jurczak, A., Koscielniak, D., Bystrowska, B., Skalniak, A. 2014. The virulence of Streptococcus mutans and the ability to form biofilms. European Journal of Clinical Microbiology \& Infectious Diseases 33(4): 499-515.

Lau, D., Plotkin, B. 2013. Antimicrobial and biofilm effects of herbs used in traditional chinese medicine. Natural Product Communications 8(11): 16171620.

Loesche, W.J. 1986. Role of Streptococcus mutans in human dental decay. Microbiological Reviews 50(4): 353-380.

Messier, C., Grenier, D. 2011. Effect of licorice compounds licochalcone A, glabridin and glycyrrhizic acid on growth and virulence properties of Candida albicans. Mycoses 54(6): e801-e806.

Messier, C., Epifano, F., Genovese, S., Grenier, D. 2012. Licorice and its potential beneficial effects in common oro-dental diseases. Oral Diseases 18(1): 32-39.

Momeni, A., Rahimian, G., Kiasi, A., Amiri, M., Kheiri, S. 2014. Effect of licorice versus bismuth on eradication of Helicobacter pylori in patients with peptic ulcer disease. Pharmacognosy Research 6(4): 341-344.

Nam, J.B., Oh, G.H., Yang, S.M., Lee, S.-E., Kang, S.-G. 2018. Evaluation of antioxidant activities of water extract from microwave torrefied oak wood. Journal of the Korean Wood Science and Technology 46(2): 178-188.

Nitalikar, M., Munde, K., Dhore, B., Shikalgar, S. 2010. Studies of antibacterial activities of Glycyrrhiza glabra root extract. International Journal of PharmTech Research 2(1): 899-901.

Park, Y.K., Lee, W.Y., Park, S.Y., Ahn, J.K., Han, M.S. 2005. Anticariogenic activity of Callistemon citrinus extract against Streptococcus mutans. Journal of the Korean Wood Science and Technology 33(2): 
72-77.

Ren, Z., Chen, L.L., Li, J.Y., Li, Y.Q. 2016. Inhibition of Streptococcus mutans polysaccharide synthesis by molecules targeting glycosyltransferase activity. Journal of Oral Microbiology 8: 31095.

Rodino, S., Butu, A., Butu, M., Cornea, P.C. 2015. Comparitive studies on antibacterial activity of licorice, elderberry and dandelion. Digest Journal of Nanomaterials and Biostructures 10(3): 947-955.

Rohinishree, Y.S., Negi, P.S. 2016. Effect of licorice extract on cell viability, biofilm formation and exotoxin production by Staphylococcus aureus. Journal of Food Science and Technology 53(2): 1092-1100.

Rosan, B., Lamont, R.J. 2000. Dental plaque formation. Microbes and Infection 2(13): 1599-1607.

Scannapieco, F.A. 1999. Role of oral bacteria in respiratory infection. Journal of Periodontology 70(7): 793-802.

Segal, R., Pisanty, S., Wormser, R., Azaz, E., Sela, M.N. 1985. Anticariogenic activity of licorice and glycyrrhizine I: Inhibition of in vitro plaque formation by Streptococcus mutans. Journal of Pharmaceutical Sciences 74(1): 79-81.

Seleem, D., Benso, B., Noguti, J., Pardi, V., Murata, R.M. 2016. In vitro and in vivo antifungal activity of lichochalcone-A against Candida albicans biofilms. PLoS ONE 11(6): e0157188.

Sharma, H., Yunus, G., Agrawal, R., Kalra, M., Verma, S., Bhattar, S. 2016. Antifungal efficacy of three medicinal plants Glycyrrhiza glabra, Ficus religiosa, and Plantago major against oral Candida albicans: A comparative analysis. Indian Journal of Dental Research 27(4): 433-436.

Shen, H., Zeng, G., Sun, B., Cai, X., Bi, L., Tang, G., Yang, Y. 2015. A polysaccharide from Glycyrrhiza inflata licorice inhibits proliferation of human oral cancer cells by inducing apoptosis via mitochondrial pathway. Tumor Biology 36(6): 4825-4831.

Shin-ichi, T., Jacynthe, D., Chantal, B., Stefan, G., Jacquelyn, R.V., Daniel, G. 2012. Reduction of bacterial volatile sulfur compound production by licoricidin and licorisoflavan A from licorice. Journal of Breath Research 6(1): 016006.

Touyz, L.Z.G. 2009. Liquorice health check, oro-dental implications, and a case report. Case Reports in Medicine 2009: 170735.

Wang, J., Chen, X., Wang, W., Zhang, Y., Yang, Z., Jin, Y., Ge, H.M., Li, E., Yang, G. 2013. Glycyrrhizic acid as the antiviral component of Glycyrrhiza uralensis Fisch. against coxsackievirus A16 and enterovirus 71 of hand foot and mouth disease. Journal of Ethnopharmacology 147(1): 114-121.

Wang, L., Yang, R., Yuan, B., Liu, Y., Liu, C. 2015. The antiviral and antimicrobial activities of licorice, a widely-used Chinese herb. Acta pharmaceutica Sinica B 5(4): 310-315.

Wang, Y.C., Yang, Y.S. 2007. Simultaneous quantification of flavonoids and triterpenoids in licorice using HPLC. Journal of Chromatography B 850(1-2): 392-399.

Welin-Neilands, J., Svensater, G. 2007. Acid tolerance of biofilm cells of Streptococcus mutans. Applied and Environmental Microbiology 73(17): 56335638.

Yu, I.C., Tsai, Y.-F., Fang, J.-T., Yeh, M.-M., Fang, J.-Y., Liu, C.-Y. 2016. Effects of mouthwash interventions on xerostomia and unstimulated whole saliva flow rate among hemodialysis patients: A randomized controlled study. International Journal of Nursing Studies 63: 9-17. 